\title{
The Super-Toda Lattice Hierarchy
}

By

\author{
Kaoru IKEDA*
}

\begin{abstract}
The super-Toda lattice (STL) hierarchy is introduced. The equivalence between the Lax representation and Zakharov-Shabat representation of the STL hierarchy is shown. Introducing the Lie superalgebra $\operatorname{osp}(\infty \mid \infty)$, the ortho-symplectic (OSp)-STL hierarchy is defined as well. These equations are solved through the Riemann-Hilbert decomposition of corresponding infinite dimensional Lie supergroups. An explicit representation of solutions is given by means of the super- $\tau$ field.
\end{abstract}

\section{§0. Introduction}

In this paper we consider the super-Toda lattice (STL) hierarchy. This paper is a complete version of our previous announcement [4], containing some new results.

The STL equations are considered in several ways. V. A. Andreyev [1] and M. A. Olshanetsky [9] classified the STL equations according to Lie superalgebras and solved them by inverse scattering method.

Inspired by the studies of the super-KP hierarchy by Yu. I. Manin and A. O. Radul [7], M. Mulase [8], K. Ueno, H. Yamada and K. Ikeda [12], [13], [14], [15], we try to investigate the STL equation by a method which is different from [1] and [9]. We extend the Toda lattice (TL) hierarchy, which is introduced by $\mathrm{K}$. Ueno and $\mathrm{K}$. Takasaki in [11], to a supersymmetric one. Considering the STL hierarchy, we can naturally extend several concepts in the theory of soliton equations, namely, the tau $(\tau)$ function and the reduction of solutions.

We first define the STL hierarchy through the Lax representation and show the equivalence with the Zakharov-Shabat representation. From the STL hierarchy, we derive the STL equation,

$$
\tilde{D}_{1}^{+} \tilde{D}_{1}^{-} u(s)=\exp (u(s)-u(s-1))+\exp (u(s+1)-u(s))
$$

where $\tilde{D}_{1}^{ \pm}$are certain odd derivations. Introducing an infinite dimensional Lie superalgebra $\operatorname{osp}(\infty \mid \infty)$ (cf. [5], [6]), we define the ortho-symplectic (OSp)-STL hierarchy. The STL equation derived from the OSp-STL hierarchy, the OSp-STL equation, reduces to the STL equation studied in [1] by adding the condition of " $4 N$-periodicity". Putting $N=1$ the OSp-STL equation is simplified to the super-sine-Gordon equation. To solve a Cauchy problem of the STL hierarchy, we consider a Riemann-Hilbert (R-H) decomposition of the Lie supergroup $\operatorname{SGL}(S)$, where $S$ is an algebra of superfields.

Communicated by M. Kashiwara, December 16, 1988. Revised March 13, 1989.

* Department of Mathematics, Tokyo Metropolitan University, Tokyo 158, Japan. 
Through the $\mathrm{R}-\mathrm{H}$ decomposition we represent components of the wave matrices of the STL hierarchy in terms of the super- $\tau$ field. Considering the $\mathrm{R}-\mathrm{H}$ decomposition for constant matrices of the Lie supergroup $\operatorname{OSp}(S)$, we solve the OSp-STL hierarchy. In his recent paper [10], Takasaki determines the equations satisfied by the super- $\tau$ field of the super-KP hierarchy in terms of the differential algebra generated by the coefficients of the wave operator of the super- $\mathbb{K} \mathbb{P}$ hierarchy. It is an interesting problem to find out the equations for our super- $\tau$ field, applying his idea.

This paper is organized as follows. In Section 1, we review shortly the theory of the TL hierarchy according to [11]. In Section 2, we define the STL hierarchy and show that the Lax representation of the STL hierarchy is equivalent to that of Zakharov-Shabat (Z-S)'s. We discuss the STL equation derived from the STL hierarchy. We also derive the ordinary TL hierarchy by taking the body part of the STL hierarchy. In Section 3, we introduce an infinite dimensional Lie superalgebra $\operatorname{osp}(\infty \mid \infty)$ and define the OSp-STL hierarchy. We consider several equations reduced from OSp-STL hierarchy. We derive the BTL and CTL hierarchy [11] by taking the body part of the OSp-STL hierarchy. In Section 4, we solve the STL hierarchy by the $\mathrm{R}-\mathrm{H}$ decomposition and give a representation of the solutions in terms of the super- $\tau$ field. We also discuss the $\mathrm{R}-\mathrm{H}$ decomposition of the OSp-STL hierarchy.

\section{Acknowledgement}

I am grateful to Professor Kimio Ueno and Dr. Takashi Takebe for fruitful discussions. I also thank Dr. Hirofumi Yamada for careful reading of the manuscript.

\section{\$1. Review of the TL Hierarchy}

Let $\Lambda=\left(\delta_{i+1, j}\right)_{i, j \in \mathbb{Z}}$ be the $\mathbb{Z} \times \mathbb{Z}$ shift matrix. We denote by $\operatorname{diag}[a(s)]$ the

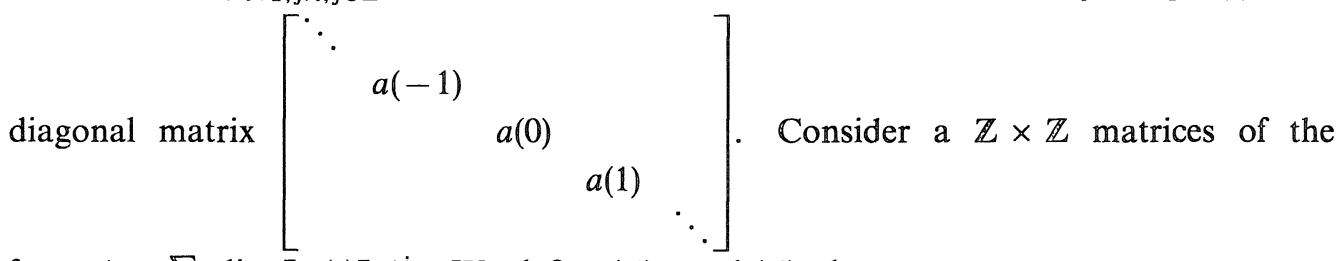
form $A=\sum_{j \in \mathbf{Z}} \operatorname{diag}\left[a_{j}(s)\right] \Lambda^{j}$. We define $(A)_{+}$and $(A)_{-}$by

$$
(A)_{+}=\sum_{j \geq 0} \operatorname{diag}\left[a_{j}(s)\right] \Lambda^{j}, \quad(A)_{-}=\sum_{j<0} \operatorname{diag}\left[a_{j}(s)\right] \Lambda^{j} .
$$

We call $(A)_{+}$and $(A)_{-}$the plus (+)-part and the minus (-)-part of $A$, respectively.

We introduce infinitely many time variables $t^{+}=\left(t_{1}^{+}, t_{2}^{+}, \ldots\right)$ and $t^{-}=\left(t_{1}^{-}, t_{2}^{-}, \ldots\right)$. Put $\mathscr{K}$ be the quotient field of $\mathbb{C}\left[\left[t^{+}, t^{-}\right]\right]$. The Toda lattice (TL) hierarchy is defined as follows. Let $L$ and $M$ be $\mathbb{Z} \times \mathbb{Z}$ matrices of the form $L=\sum_{j=0}^{\infty} \operatorname{diag}\left[u_{j}(s)\right] \mathbb{1}^{1-j}$, $M=\sum_{j=0}^{\infty} \operatorname{diag}\left[v_{j}(s)\right] \Lambda^{-1+j}$, where $u_{j}(s), v_{j}(s) \in \mathscr{K}, u_{0}(s)=1$ and $v_{0}(s) \neq 0$. The $\mathbb{T L}$ hierarchy is the system of infinitely many Lax equations: 


$$
\begin{array}{ll}
\partial_{t_{n}^{+}} L=\left[B_{n}, L\right], & \partial_{t_{n}^{+}} M=\left[B_{n}, M\right], \\
\partial_{t_{n}^{-}} L=\left[C_{n}, L\right], & \partial_{t_{n}^{-}} M=\left[C_{n}, M\right],
\end{array}
$$

where $B_{n}=\left(L^{n}\right)_{+}$and $C_{n}=\left(M^{n}\right)_{-}$.

Theorem 1.1 ([11]). The TL hierarchy (1.1) is equivalent to the following system of the Zakharov-Shabat (Z-S) equations.

$$
\begin{aligned}
& \partial_{t_{n}^{+}} B_{m}-\partial_{t_{m}^{+}} B_{n}=\left[B_{n}, B_{m}\right], \\
& \partial_{t_{n}^{+}} C_{m}-\partial_{t_{m}^{-}} B_{n}=\left[B_{n}, C_{m}\right], \\
& \partial_{t_{n}^{-}} C_{m}-\partial_{t_{m}^{-}} C_{n}=\left[C_{n}, C_{m}\right] .
\end{aligned}
$$

Put $m=n=1$ in the second equation and put $B_{1}=\Lambda+\operatorname{diag}[b(s)]$, $C_{1}=\operatorname{diag}[c(s)] \Lambda^{-1}$. Let $u(s)$ be a function such that $b(s)=\partial_{t_{1}^{+}} u(s)$ and $c(s)=$ $\exp (u(s)-u(s-1))$. Then we have the two dimensional Toda lattice equation

$$
\partial_{t_{1}^{+}} \partial_{t_{1}} u(s)=\exp (u(s)-u(s-1))-\exp (u(s+1)-u(s)) \text {. }
$$

From (1.2), there exist

$$
W_{+}=\sum_{j=0}^{\infty} \operatorname{diag}\left[w_{j}^{+}(s)\right] \Lambda^{-j}, \quad W_{-}=\sum_{j=0}^{\infty} \operatorname{diag}\left[w_{j}^{-}(s)\right] \Lambda^{j},
$$

with $w_{j}^{ \pm}(s) \in \mathscr{K}, w_{0}^{+}(s)=1$ and $w_{0}^{-}(s) \neq 0$, satisfying

$$
\begin{aligned}
& \partial_{t_{n}^{+}} W_{+}=B_{n} W_{+}-W_{+} \Lambda^{n}, \quad \partial_{t_{n}^{+}} W_{-}=C_{n} W_{-}, \\
& \partial_{t_{n}^{-}} W_{+}=C_{n} W_{+}, \quad \partial_{t_{n}^{-}} W_{-}=C_{n} W_{-}-W_{-} \Lambda^{-n},
\end{aligned}
$$

where $B_{n}=\left(W_{+} \Lambda^{n} W_{+}^{-1}\right)_{+}, C_{n}=\left(W_{-} \Lambda^{-n} W_{-}^{-1}\right)_{-}$. Conversely the existence of $W_{ \pm}$satisfying (1.3) implies (1.2) as a compatibility condition of (1.3). We call (1.3) the Sato equations and $W_{ \pm}$the wave matrices of the TL hierarchy.

To solve the TL hierarchy we introduce an infinite dimensional Lie group $G L(\infty ; \mathscr{K})$ as follows.

$$
G L(\infty ; \mathscr{K})=\{A \in \operatorname{Mat}(\mathbf{Z} \times \mathbf{Z} ; \mathscr{K}) \mid A \text { is invertible. }\}
$$

Note that $W_{ \pm} \in G L(\infty ; \mathscr{K})$. Put $\Phi_{+}=\Phi_{+}\left(t^{+}\right)=\exp \left(\sum_{j=1}^{\infty} t_{j}^{+} \Lambda^{j}\right) \quad$ and $\quad \Phi_{-}\left(t^{-}\right)=$ $\exp \left(\sum_{j=1}^{\infty} t_{j}^{-} \Lambda^{-j}\right)$. We denote by $G L(\infty ; \mathbf{C})$ the subgroup of $G L(\infty ; \mathscr{K})$ consisting of constant matrices.

Theorem 1.2 ([11]). Suppose that, for $A \in G L(\infty ; C)$, the following decomposition with $W_{ \pm}$is satisfied:

$$
\Phi_{+} A \Phi_{-}^{-1}=W_{+}^{-1} W_{-} .
$$

Then $W_{ \pm}$are the wave matrices of the TL hierarchy.

We call (1.4) the Riemann-Hilbert $(\mathrm{R}-\mathrm{H})$ decomposition. Put $H=\Phi_{+} A \Phi_{-}=$ 
$\left(h_{i j}\right)_{i, j \in \mathbb{Z} .}$ From (1.4) we see that $\left(W_{+} H\right)_{-}=0$. This induces the linear equation

$$
\left(\ldots w_{2}^{+}(s), w_{1}^{+}(s), 1\right) \mathbb{M}(s)=(\ldots 0,0,0)
$$

for all $s \in \mathbb{Z}$, where $M(s)=\left(h_{s-i+1, s-j}\right)_{i, j<0}$. Put $\tau(s)=\operatorname{det}\left(h_{s-i, s-j}\right)_{i, j>0}$. Then we have the following explicit representation.

Thleorem $\mathbb{1} 3([11]) . \quad$ (i) $w_{j}^{+}(s)=\frac{p_{j}\left(-\tilde{\partial}_{+}\right) \tau(s)}{\tau(s)}$,

(ii) $w_{j}^{-}(s)=\frac{p_{j}\left(-\tilde{\partial}_{-}\right) \tau(s+1)}{\tau(s)}$

where $p_{j}\left(t_{1}, t_{2}, \ldots\right)$ is defined by $\exp \left(\sum_{j=1}^{\infty} t_{j} k^{j}\right)=\sum_{j=0}^{\infty} p_{j}\left(t_{1}, t_{2}, \ldots\right) k^{j}$ and $\tilde{\partial}_{ \pm}=$ $\left(\partial_{t_{1}^{ \pm}}, 2^{-1} \partial_{t_{2}^{ \pm}}, 3^{-1} \partial_{t_{3}^{ \pm}}, \ldots\right)$.

We define the matrices $J=\left((-)^{i} \delta_{i,-j}\right)_{i, j \in \mathbb{Z}}$ and $K=\Lambda J$. We introduce the infinite dimensional Lie algebras

$$
\begin{aligned}
o(\infty ; \mathscr{K}) & =\left\{A \in \operatorname{Mat}(\mathbb{Z} \times \mathbb{Z} ; \mathscr{K}) \mid J^{t} A+A J=0\right\}, \\
s p(\infty ; \mathscr{K}) & =\left\{A \in \mathbb{M a t}(\mathbb{Z} \times \mathbb{Z} ; \mathscr{K}) \mid \mathbb{K} A+{ }^{t} A \mathbb{K}=0\right\} .
\end{aligned}
$$

The corresponding Lie groups are

$$
\begin{aligned}
O(\infty ; \mathscr{K}) & =\left\{A \in \operatorname{Mat}(\mathbb{Z} \times \mathbb{Z} ; \mathscr{K}) \mid J^{t} A J=A^{-1}\right\}, \\
S p(\infty ; \mathscr{K}) & =\left\{A \in \operatorname{Mat}(\mathbb{Z} \times \mathbb{Z} ; \mathscr{K}) \mid{ }^{t} K^{t} A K=A^{-1}\right\} .
\end{aligned}
$$

Put $t^{ \pm}{ }_{2 n}=0$ for $n=1,2, \ldots$ in (1.3). By adding symmetry conditions to the wave matrices so that $W_{ \pm} \in O(\infty ; \mathscr{K})$ (resp. $S p(\infty ; \mathscr{K})$ ), we obtain the $\mathbb{B T L}$ (resp. CTL) hierarchy.

Remark. Assume that $W_{+}$are the wave matrices of the $\mathbb{B T L}$ (resp. CTL) hierarchy. Then $B_{n}=\left(W_{+} \Lambda^{n} W_{+}^{-1}\right)_{+}$and $C_{n}=\left(W_{-} \Lambda^{-n} W_{-}^{-1}\right)_{-} \in o(\infty ; \mathscr{K})(\operatorname{resp} . \operatorname{sp}(\infty ; \mathscr{K}))$ for odd $n$.

Theorem $\mathbb{1} .4([11])$. Let $t^{ \pm}{ }_{2 n}=0$ for $n=1,2, \ldots$. Assume that $A \in O(\infty ; \mathbb{C})$ (resp. $S p(\infty ; \mathbb{C})$ in (1.4). Then $W_{ \pm} \in O(\infty ; \mathscr{K})$ (resp. $\left.S p(\infty ; \mathscr{K})\right)$, namely $W_{ \pm}$are the wave matrices of the $\mathrm{BTL}$ (resp. CTL) hierarchy.

\section{\$2. The STL Hierarch}

Throughout this paper, $\underline{k}$ denotes the modulo class of $k$ by $\mathbb{Z}_{2}$. Let $\mathscr{A}=\mathscr{A}_{0} \oplus \mathscr{A}_{1}$ be an arbitrary supercommutative superalgebra. The body map is the canonical projection $\varepsilon: \mathscr{A} \rightarrow \mathscr{A} /\left(\mathscr{A}_{1}\right)$, where $\left(\mathscr{A}_{1}\right)$ is the ideal generated by $\mathscr{A}_{\underline{1}}$. For $a=a_{\underline{0}}+a_{\underline{1}} \in \mathscr{A}$, $a_{i} \in \mathscr{A}_{i}$, we denote $a^{*}=a_{0}-a_{1}$. The body map $\varepsilon$ and the operator $*$ can be naturally extended on the superalgebra of matrices with entries in $\mathscr{A}$. Namely, for $A=\left(a_{i j}\right)_{i, j}\left(a_{i j} \in\right.$ $\mathscr{A}), \varepsilon(A)=\left(\varepsilon\left(a_{i j}\right)\right)_{i, j}$ and $A^{*}=\left(a_{i j}^{*}\right)_{i, j}$. For $k \in \mathbb{Z}$, put $A^{*(k)}=A(\underline{k}=0),=A^{*}(\underline{k}=1)$. 
Moreover we put, for $n \geq 1, A_{*}^{n}=A^{*(n-1)} A_{*}^{n-1}, A_{*}^{1}=A$. If $D$ is an odd derivation acting on $\mathscr{A}$, then it satisfies the super-Leibniz rule:

$$
D(a b)=(D a) b+a^{*}(D b), \quad a, b \in \mathscr{A} .
$$

Let $V$ be the Grassmann algebra $\Lambda\left(\mathbb{C}^{\infty}\right)$ with infinitely many generators, $e_{1}, e_{2}, \ldots$ Let $t^{+}=\left(t_{1}^{+}, t_{2}^{+}, \ldots\right)$ and $t^{-}=\left(t_{1}^{-}, t_{2}^{-}, \ldots\right)$ be infinitely many Grassmann variables. Here $t_{2 j}^{ \pm}$are even (commutative) variables and $t_{\frac{1}{2 j}-1}^{ \pm}$are odd (anti-commutative) ones. Let $\mathscr{K}$ be the quotient field of the $\mathbb{C}$-algebra $\mathbb{C}\left[\left[t_{2}^{\frac{t}{2}}, t_{4}^{ \pm}, \ldots\right]\right]$. The supercommutative superalgebra $S=S_{0} \oplus S_{1}$ is now defined by

$$
S=\mathscr{K} \otimes \mathbb{C}\left[\left[t_{1}^{ \pm}, t_{3}^{ \pm}, \ldots\right]\right] \otimes V .
$$

We define the super-vector fields, acting on $S$,

$$
D_{\frac{2 j}{j}}^{ \pm}=\partial_{t_{2 j}^{ \pm}}, \quad D_{2 j-1}^{ \pm}=\partial_{t_{2 j-1}^{ \pm}}+\sum_{k \geq 1} t_{\frac{1}{2 k-1}}^{\frac{ \pm}{2}} \partial_{t_{2 j}^{ \pm}+2 k-2}, \quad j \geq 1
$$

These super-vector fields satisfy the (anti-) commutation relations

$$
\left[D_{j}^{ \pm}, D_{k}^{ \pm}\right]_{(-)^{j k+1}}=2 \delta_{\underline{j k}, \underline{1}} D_{j+k}^{ \pm}, \quad\left[D_{j}^{ \pm}, D_{k}^{\mp}\right]_{(-)^{j k+1}}=0,
$$

where []$_{1}=[]_{+}$(=anti-commutator) and []$_{-1}=[]$ (=commutator). Now we give the definition of the super-Toda lattice (STL) hierarchy. Define the matrices $L$ and $M$ as follows:

$$
\begin{aligned}
L & =\sum_{j=0}^{\infty} \operatorname{diag}\left[u_{j}(s)\right] \Lambda^{1-j}, \quad \text { with } \quad u_{0}(s)=1 \quad \text { and } \quad u_{j}(s) \in S_{\underline{j}} . \\
M & =\sum_{j=0}^{\infty} \operatorname{diag}\left[v_{j}(s)\right] \Lambda^{-1+j}, \quad \text { with } \quad \varepsilon\left(v_{0}(s)\right) \neq 0 \quad \text { and } \quad v_{j}(s) \in S_{\underline{j}} .
\end{aligned}
$$

We put $B_{n}=\left(L_{*}^{n}\right)_{+}, C_{n}=\left(M_{*}^{n}\right)_{-}$. The STL hierarchy is a system of equations of the Lax type,

$$
\begin{aligned}
D_{m}^{+} L & =(-)^{m} B_{m}^{*} L-L^{*(m)} B_{m}+2 \delta_{\underline{m}, \underline{1}} L_{*}^{m+1}, \\
D_{m}^{+} M & =(-)^{m} B_{m}^{*} M-M^{*(m)} B_{m}, \\
D_{m}^{-} L & =(-)^{m} C_{m}^{*} L-L^{*(m)} C_{m}, \\
D_{m}^{-} M & =(-)^{m} C_{m}^{*} M-M^{*(m)} C_{m}+2 \delta_{\underline{m}, \underline{1}} M_{*}^{m+1} .
\end{aligned}
$$

Theorem 2.1. The STL hierarchy (2.1) (2.4) is equivalent to the system of the Zakharov-Shabat (Z-S) type:

$$
\begin{gathered}
D_{n}^{+} C_{m}-(-)^{m n} D_{m}^{-} B_{n}=(-)^{m n} B_{n}^{*(m)} C_{m}-C_{m}^{*(n)} B_{n} . \\
D_{m}^{+} B_{n}-(-)^{m n} D_{n}^{+} B_{m}=(-)^{m n} B_{m}^{*(n)} B_{n}-B_{n}^{*(m)} B_{m}+2 \delta_{\underline{m n}, \underline{1}} B_{m+n}, \\
D_{m}^{-} C_{n}-(-)^{m n} D_{n}^{-} C_{m}=(-)^{m n} C_{m}^{*(n)} C_{n}-C_{n}^{*(m)} C_{m}+2 \delta_{m n, 1} C_{m+n} .
\end{gathered}
$$

Proof. First we show that the Lax type system $((2.1) \sim(2.4))$ induces the Z-S type system $((2.5) \sim(2.7))$. We can easily see that 


$$
\begin{gathered}
D_{m}^{+} L_{*}^{n}=(-)^{m n} B_{m}^{*(n)} L_{*}^{n}-L_{*}^{n *(m)} B_{m}+2 \delta_{\underline{m n}, \underline{1}} L_{*}^{m+n}, \\
D_{m}^{+} M_{*}^{n}=(-)^{m n} B_{m}^{*(n)} M_{*}^{n}-M_{*}^{n *(m)} B_{m}, \\
D_{m}^{-} L_{*}^{n}=(-)^{m n} C_{m}^{*(n)} L_{*}^{n}-L_{*}^{n *(m)} C_{m}, \\
D_{m}^{-} M_{*}^{n}=(-)^{m n} C_{m}^{*(n)} M_{*}^{n}-M_{*}^{n *(m)} C_{m}+2 \delta_{\underline{m n}, \underline{1}} M_{*}^{m+n} . \\
(m, n=1,2,3, \ldots)
\end{gathered}
$$

Taking the (+)-part of (2.8), we have

Using the identity

$$
\begin{aligned}
D_{m}^{+} B_{n}-(-)^{m n} D_{n}^{+} B_{m}= & 2(-)^{m n} B_{m}^{*(n)} B_{n}-2 B_{n}^{*(m)} B_{m}+(-)^{m n}\left(B_{m}^{*(n)}\left(L_{*}^{n}\right)_{-}\right)_{+} \\
& -\left(\left(L_{*}^{n}\right)_{-}^{*(m)} B_{m}\right)_{+}-\left(B_{n}^{*(m)}\left(L_{*}^{m}\right)_{-}\right)_{+} \\
& +(-)^{m n}\left(\left(L_{*}^{m}\right)_{-}^{*(n)} B_{n}\right)_{+}+4 \delta_{m n, 1} B_{m+n} .
\end{aligned}
$$

we have

$$
L_{*}^{n *(m)} L_{*}^{m}-(-)^{m n} L_{*}^{m *(n)} L_{*}^{n}=2 \delta_{\underline{m n}, \underline{1}} L_{*}^{m+n}
$$

$$
\begin{aligned}
& \left(B_{n}^{*(m)}\left(L_{*}^{m}\right)_{-}\right)_{+}+\left(\left(L_{*}^{n}\right)_{-}^{*(m)} B_{m}\right)_{+}-(-)^{m n}\left(B_{m}^{*(n)}\left(L_{*}^{n}\right)_{-}\right)_{+}-(-)^{m n}\left(\left(L_{*}^{m}\right)_{-}^{*(n)} B_{n}\right)_{+} \\
& \quad=-B_{n}^{*(m)} B_{m}+(-)^{m n} B_{m}^{*(n)} B_{n}+2 \delta_{\underline{m n}, \underline{1}} B_{m+n} .
\end{aligned}
$$

Then (2.12) reduces to (2.6). From (2.9) and (2.10), we have

$$
\begin{aligned}
D_{m}^{+} M_{*}^{n} & =(-)^{m n} B_{m}^{*(n)} M_{*}^{n}-M_{*}^{n *(m)} B_{m}, \\
D_{n}^{-} L_{*}^{m} & =(-)^{m n} C_{n}^{*(m)} L_{*}^{m}-L_{*}^{m *(n)} C_{n} .
\end{aligned}
$$

We denote by $(Y)_{-}$and $(Y)_{+}$the $(-)$-part of $(\Upsilon)$ and the $(+)$-part of $(Y)$. By taking the difference $(Y)_{-}-(-)^{m n}(Y)_{+}$we obtain (2.5). We obtain (2.7) similarly.

Now we show the converse.

Lemma 2.2. The matrices $L, M$ satisfy the following equations:

$$
\begin{aligned}
& D_{m}^{+} L_{*}^{n}-(-)^{m n} B_{m}^{*(n)} L_{*}^{n}+L_{*}^{n *(m)} B_{m}-2 \delta_{\underline{m n}, \underline{1}} L_{*}^{m+n} \\
& \quad=\sum_{l=0}^{n-1}(-)^{(l+n+1) m} L_{*}^{l *(m+n+l)}\left(D_{m}^{+} L-(-)^{m} B_{m}^{*} L+L^{*(m)} B_{m}-2 \delta_{\underline{m}, \underline{1}} L_{*}^{m+1}\right)^{*(l+n+1)} L_{*}^{n-1-l}, \\
& D_{m}^{-} L_{*}^{n}-(-)^{m n} C_{m}^{*(n)} L_{*}^{n}+L_{*}^{n *(m)} C_{m} \\
& \quad=\sum_{l=0}^{n-1}(-)^{m(l+n+1)} L_{*}^{l *(m+n+l)}\left(D_{m}^{-} L-(-)^{m} C_{m}^{*} L+L^{*(m)} C_{m}\right)^{*(l+n+1)} L_{*}^{n-l-1}, \\
& D_{m}^{+} M_{*}^{n}-(-)^{m n} B_{m}^{*(n)} M_{*}^{n}+M_{*}^{n *(m)} B_{m} \\
& \quad=\sum_{l=0}^{n-1}(-)^{m(l+n+1)} M_{*}^{l *(m+l+n)}\left(D_{m}^{+} M-(-)^{m} B_{m}^{*} M+M^{*(m)} B_{m}\right)^{*(l+n+1)} M_{*}^{n-1-l}, \\
& D_{m}^{-} M_{*}^{n}-(-)^{m n} C_{m}^{*(n)} M_{*}^{n}+M_{*}^{n *(m)} C_{m}-2 \delta_{\underline{m n}, \underline{1}} M_{*}^{m+n} \\
& \quad=\sum_{l=0}^{n-1}(-)^{(l+n+1) m} M_{*}^{l *(m+n+l)}\left(D_{m}^{-} M-(-)^{m} C_{m}^{*} M+M^{*(m)} B_{m}\right. \\
& \left.\quad-2 \delta_{\underline{m}, \underline{1}} M_{*}^{m+1}\right)^{*(l+n+1)} M_{*}^{n-1-l} .
\end{aligned}
$$


Proof. First we show (2.14) by induction. It holds trivially for $n=1$. For $n \geq 0$,

$$
\begin{aligned}
D_{m}^{+} & L_{*}^{n+1}-(-)^{(n+1) m} B_{m}^{*(n+1)} L_{*}^{n+1}+L_{*}^{n+1 *(m)} B_{m}-2 \delta_{\underline{m(n+1)}, \underline{1}} L_{*}^{m+n+1} \\
= & (-)^{m n}\left(D_{m}^{+} L-(-)^{m} B_{m}^{*} L+L^{*(m)} B_{m}-2 \delta_{\underline{m}, \underline{1}} L_{*}^{m+1}\right)^{*(n)} L_{*}^{n} \\
& +L^{*(m+n)}\left(D_{m}^{+} L_{*}^{n}-(-)^{m n} B_{m}^{*(n)} L_{*}^{n}+L_{*}^{n *(m)} B_{m}-2 \delta_{\underline{m n}, \underline{1}} L_{*}^{m+n}\right) \\
& +2\left((-)^{m n} \delta_{\underline{m}, \underline{1}}+\delta_{\underline{m n}, \underline{1}}-\delta_{\underline{m(n+1)}, \underline{1}}\right) L_{*}^{m+n+1} .
\end{aligned}
$$

Notice that $(-)^{m n} \delta_{\underline{m}, \underline{1}}+\delta_{\underline{m n}, \underline{1}}-\delta_{\underline{m(n+1), 1}}=0 . \quad$ By the induction hypothesis we have the conclusion. We can show $(2.15) \sim(2.17)$ similarly.

We define ord $A$ and coord $A$ for $A=\sum_{j \in \mathbf{Z}} \operatorname{diag}\left[a_{j}(s)\right] \Lambda^{j}$ as follows:

$$
\begin{aligned}
\operatorname{ord} A & =\inf \left\{j \in \mathbb{Z} \mid \operatorname{diag}\left[a_{i}(s)\right]=0, \text { for } i>j\right\}, \\
\operatorname{coord} A & =\sup \left\{j \in \mathbb{Z} \mid \operatorname{diag}\left[a_{i}(s)\right]=0, \text { for } i<j\right\} .
\end{aligned}
$$

Lemma 2.3. Suppose that $D_{m}^{+} L-(-)^{m} B_{m}^{*} L+L^{*(m)} B_{m}-2 \delta_{\underline{m n}, \underline{1}} L_{*}^{m+1}=\operatorname{diag}[a(s)] \Lambda^{r}+$ lower order, for some $r$ and $\operatorname{diag}[a(s)] \neq 0$. Then

$$
\lim _{n \rightarrow \infty} \operatorname{ord}\left(D_{m}^{+} L_{*}^{n}-(-)^{m n} B_{m}^{*(n)} L_{*}^{n}+L_{*}^{n *(m)} B_{m}-2 \delta_{\underline{m n}, \underline{1}} L_{*}^{m+n}\right)=+\infty .
$$

Proof. By using Lemma 2.2, we see that the highest order term of the left hand side of (2.14) is

$$
\operatorname{diag}\left[\sum_{l=0}^{n-1}(-)^{(l+n+1)(1+r)} a(s+l)\right] \Lambda^{r+n-1} .
$$

Fact A. For any $n \in \mathbf{N}$, there exists $n^{\prime} \in \mathbf{N}$ such that $n^{\prime}>n$ and

$$
\operatorname{diag}\left[\sum_{l=0}^{n^{\prime}-1}(-)^{\left(l+n^{\prime}+1\right)(1+r)} a(s+l)\right] \neq 0 \text {. }
$$

Proof of Fact $A$. Suppose that there exists $N \in \mathbb{N}$ such that

$$
\begin{gathered}
\sum_{l=0}^{N-1}(-)^{(l+N+1)(1+r)} a(s+l)=0 \quad \text { and } \\
\sum_{l=0}^{N}(-)^{(l+N)(1+r)} a(s+l)=0, \quad \text { for all } s \in \mathbf{Z} .
\end{gathered}
$$

From this we have $a(s+N)=0$ for all $s \in \mathbb{Z}$. This contradicts the assumption of Lemma 2.3.

By Fact A the highest order term of the left hand side of (2.14) never vanishes as $n \rightarrow \infty$. This completes the proof of Lemma 2.3.

On the other hand we see the following fact.

Fact B. The order of the left hand side of (2.14) is less than $m$. 
Proof of Fact B. From (2.6) we see that

$$
\begin{aligned}
& D_{m}^{+} L_{*}^{n}-(-)^{m n} B_{m}^{*(n)} L_{*}^{n}+L_{*}^{n *(m)} B_{m}-2 \delta_{\underline{m n}, \underline{1}} L_{*}^{m+n} \\
& \quad=D_{m}^{+}\left(L_{*}^{n}\right)_{-}+(-)^{m n} D_{n}^{+} B_{m}-(-)^{m n} B_{m}^{*(n)}\left(L_{*}^{n}\right)_{-}+\left(L_{*}^{n *(m)}\right)_{-} B_{m}-2 \delta_{\underline{m n}, \underline{1}}\left(L_{*}^{m+n}\right)_{-} .
\end{aligned}
$$

The order of the right hand side of (2.19) is less than $m$.

From Lemma 2.5 and Fact $\mathbb{B}$, we can conclude that

$$
D_{m}^{+} L-(-)^{m} B_{m}^{*} L+L^{*(m)} B_{m}-2 \delta_{\underline{m}, \underline{1}} L_{*}^{m+1}=0 .
$$

Secondly we show (2.2).

Lemm2 2.4. Suppose that $\mathbb{D}_{m}^{+} M-(-)^{m} B_{m}^{*} M+M^{*(m)} B_{m}=\operatorname{diag}[b(s)] \Lambda^{r}+$ higher order terms for some $r$ and $\operatorname{diag}[b(s)] \neq 0$. Then

$$
\lim _{n \rightarrow \infty} \operatorname{coord}\left(D_{m}^{+} M_{*}^{n}-(-)^{m n} B_{m}^{*(n)} M_{*}^{n}+M_{*}^{n *(m)} B_{m}\right)=-\infty .
$$

Proof. From Lemma 2.2 we see that the lowest order term of the left hand side of (2.16) is

$$
\operatorname{diag}\left[\sum_{l=0}^{n-1}(-)^{(l+n+1)(r+1)} v_{0}(s) \ldots v_{0}(s-l+1) v_{0}(s+r-1) \ldots v_{0}(s-n+2+r) b(s-1)\right] \Lambda^{-n+1+r} .
$$

Fact $\mathbb{C}$. For any $n \in \mathbb{N}$, there exists $n^{\prime} \in \mathbb{N}$ such that $n^{\prime} \geq n$ and

$$
\operatorname{diag}\left[\sum_{l=0}^{n^{\prime}-1}(-)^{\left(l+n^{\prime}+1\right)(r+1)} v_{0}(s) \ldots v_{0}(s-l+1) v_{0}(s+r-1) \ldots v_{0}\left(s-n^{\prime}+2+r\right) b(s-l)\right] \neq 0 .
$$

The proof is similar to that of Fact A. By Fact C, the lowest order term of the left hand side of (2.16) never vanishes. This completes the proof of Lemma 2.4 .

Fact D. The coorder of the left hand side of (2.16) is positive.

Proof of Fact D. From (2.5) we see that

$$
\begin{aligned}
& D_{m}^{+} M_{*}^{n}-(-)^{m n} B_{m}^{*(n)} M_{*}^{n}+M_{*}^{n *(m)} B_{m} \\
& \quad=D_{m}^{+}\left(M_{*}^{n}\right)_{+}+(-)^{m n} D_{n}^{-} B_{m}-(-)^{m n} B_{m}^{*(n)}\left(M_{*}^{n}\right)_{+}+\left(M_{*}^{n}\right)_{+}^{*(m)} B_{m} .
\end{aligned}
$$

This assures the claim of Fact $D$.

From Lemma 2.4 and Fact D, we see that

$$
D_{m}^{+} M-(-)^{m} B_{m}^{*} M+M^{*(m)} B_{m}=0 .
$$

The equations (2.3) and (2.4) can be shown similarly.

Q.E.D.

We define $\tilde{D}_{1}^{ \pm}, \widetilde{B}_{1}$ and $\tilde{C}_{1}$ as the restriction of $D_{1}^{ \pm}, B_{1}$ and $C_{1}$ to the sector $t_{\overline{2} j+1}^{t}=0, j \geq 1$, respectively. Accordingly let

$$
\tilde{D}_{1}^{ \pm}=\partial_{t_{1}^{ \pm}}+t_{1}^{ \pm} \partial_{t_{2}^{ \pm}}
$$


From (2.5), we get

$$
\tilde{D}_{1}^{+} \tilde{C}_{1}+\tilde{D}_{1}^{-} \tilde{B}_{1}=-\tilde{B}_{1}^{*} \tilde{C}_{1}-\tilde{C}_{1}^{*} \tilde{B}_{1}
$$

Substituting $\tilde{B}_{1}=\Lambda+\operatorname{diag}[\tilde{b}(s)]$ and $\tilde{C}_{1}=\operatorname{diag}[\tilde{c}(s)] \Lambda^{-1}$ to $(2.19)$, we have

$$
\begin{gathered}
\tilde{D}_{1}^{-} \tilde{b}(s)=-\tilde{c}(s)-\tilde{c}(s+1), \\
\tilde{D}_{1}^{+} \tilde{c}(s)=(\tilde{b}(s)-\tilde{b}(s+1)) \tilde{c}(s)
\end{gathered}
$$

Let $u(s) \in S_{0}$ be a superfield such that

$$
\begin{gathered}
\tilde{b}(s)=\tilde{D}_{1}^{+} u(s), \\
\tilde{c}(s)=\exp (u(s)-u(s-1)) .
\end{gathered}
$$

Then one can see that $(2.20)$ and (2.21) reduce to a single equation

$$
\widetilde{D}_{1}^{+} \widetilde{D}_{1}^{-} u(s)=\exp (u(s)-u(s-1))+\exp (u(s+1)-u(s)) .
$$

We call (2.22) the STL equation. The body part $f(s)=\varepsilon(u(s))$ satisfies the ordinary TL equation,

$$
\partial_{t_{2}^{+}} \partial_{t_{2}^{-}} f(s)=\exp (f(s)-f(s-2))-\exp (f(s+2)-f(s)) .
$$

Let $W_{+}$and $W_{-}$be matrices such that

$$
W_{+}=\sum_{j=0}^{\infty} \operatorname{diag}\left[w_{j}^{+}(s)\right] \Lambda^{-j}, \quad W_{-}=\sum_{j=0}^{\infty} \operatorname{diag}\left[w_{j}^{-}(s)\right] \Lambda^{j},
$$

where $w_{0}^{+}(s)=1, \varepsilon\left(w_{0}^{-}(s)\right) \neq 0$ and $w_{j}^{ \pm}(\mathrm{s}) \in S_{j}$. From $(2.5) \sim(2.7)$ we can conclude the existence of $W_{ \pm}$satisfying the equations

$$
\begin{aligned}
& D_{n}^{+} W_{+}=B_{n} W_{+}-W_{+}^{*(n)} \Lambda^{n}, \quad D_{n}^{+} W_{-}=B_{n} W_{-}, \\
& D_{n}^{-} W_{+}=C_{n} W_{+}, \quad D_{n}^{-} W_{-}=C_{n} W_{-}-W^{*(n)} \Gamma^{-n},
\end{aligned}
$$

where $\Gamma=\left((-)^{i} \delta_{i+1, j}\right)_{i, j \in \mathbf{Z}}$. Conversely the existence of $W_{ \pm}$of $(2.23)$ implies $(2.5) \sim(2.7)$ as a compatibility condition. We call (2.23) the Sato equations and $W_{ \pm}$the wave matrices of the STL hierarchy.

Finally we mention a relation with the ordinary TL hierarchy. With $A=\left(a_{i, j}\right)_{i, j \in \mathbf{Z}}$, we associate a matrix

$$
\check{A}=\left[\begin{array}{ll}
A_{00} & A_{01} \\
A_{10} & A_{11}
\end{array}\right], \quad \text { where } \quad A_{i j}=\left(a_{\mu v}\right)_{\underline{\mu}=i, \underline{v}=j} .
$$

Put $\varepsilon\left(\check{W}_{ \pm}\right)=\left[\begin{array}{cc}W_{00}^{ \pm} & 0 \\ 0 & W_{11}^{ \pm}\end{array}\right]$for the wave matrices of the STL hierarchy. Then $W_{00}^{ \pm}$and $W_{1} \frac{ \pm}{1}$ are the wave matrices of the ordinary TL hierarchy.

\section{§3. The OSp-STL Hierarchy}

In this section, we investigate the OSp-STL hierarchy. Consider the Lie superalgebra $g l_{\underline{0}}(\infty \mid \infty)=g l_{\underline{0}}(\infty \mid \infty) \oplus g l_{\underline{1}}(\infty \mid \infty)$, 


$$
\begin{aligned}
& g l_{\underline{0}}(\infty \mid \infty)=\left\{\left[\begin{array}{cc}
A_{00} & 0 \\
0 & A_{11}
\end{array}\right] ; A_{00}, A_{11} \in \operatorname{Mat}(\mathbb{Z} \times \mathbb{Z}: \mathbb{C})\right\}, \\
& g l_{\underline{1}}(\infty \mid \infty)=\left\{\left[\begin{array}{cc}
0 & A_{01} \\
A_{10} & 0
\end{array}\right] ; A_{01}, A_{10} \in \operatorname{Mat}(\mathbb{Z} \times \mathbb{Z}: \mathbb{C})\right\} .
\end{aligned}
$$

Next we define $g l(S)=g l_{\underline{0}}(S) \oplus g l_{\underline{1}}(S)$ by

$$
g l_{\underline{i}}(S)=\bigoplus_{\underline{\mu+v}=i} g l_{\underline{\mu}}(\infty \mid \infty) \otimes S_{\underline{v}} .
$$

The Lie supergroup $\operatorname{SGL}(S)$ is defined by

$\operatorname{SGL}(S)$

$$
=\left\{A=\left[\begin{array}{ll}
A_{00} & A_{01} \\
A_{10} & A_{11}
\end{array}\right] ; A_{i j}=\left(a_{i j}^{\mu v}\right)_{i, j \in \mathbb{Z}}, a_{i j}^{\mu v} \in S_{\underline{\mu+v}}, \varepsilon\left(A_{00}\right) \text { and } \varepsilon\left(A_{11}\right) \text { are invertible }\right\} .
$$

Substituting $V$ for $S$, we can similarly define $\operatorname{SGL}(V)$. We define two operators " $s t$ " and "st" on $g l(\infty \mid \infty)$ and $g l(S)$ respectively by

$$
\begin{gathered}
{ }^{s t} A={ }^{s t}\left[\begin{array}{ll}
A_{00} & A_{01} \\
A_{10} & A_{11}
\end{array}\right]=\left[\begin{array}{rr}
{ }^{t} A_{00} & { }^{t} A_{10} \\
-{ }^{t} A_{01} & { }^{t} A_{11}
\end{array}\right], \\
{ }^{\tilde{s} t} B={ }^{\tilde{s} t}\left[\begin{array}{ll}
B_{00} & B_{01} \\
B_{10} & B_{11}
\end{array}\right]=\left[\begin{array}{cc}
{ }^{t} B_{00} & (-)^{i t} B_{10} \\
(-)^{i+1}{ }^{t} B_{01} & { }^{t} B_{11}
\end{array}\right],
\end{gathered}
$$

for $A \in g l(\infty \mid \infty)$ and $B \in g l_{\underline{i}}(S)$. Note that

$$
{ }^{s t}(A B)=(-)^{i j s t} B^{s t} A, \quad{ }^{\tilde{s} t}(C D)=(-)^{i j \tilde{s} t} D^{\tilde{s} t} C
$$

for $A \in g l_{\underline{i}}(\infty \mid \infty), B \in g l_{\underline{j}}(\infty \mid \infty), C \in g l_{\underline{i}}(S)$ and $D \in g l_{\underline{j}}(S)$. We introduce a Lie superalgebra osp $(\infty \mid \infty)$ (cf [5], [6]). Put $P=\left[\begin{array}{cc}J & 0 \\ 0 & -K\end{array}\right] \in g l(\infty \mid \infty)$, with $J$ and $K$ defined in $\S 1$. The Lie superalgebra osp $(\infty \mid \infty)$ is introduced as

$$
\operatorname{osp}(\infty \mid \infty)=\left\{A \in g l(\infty \mid \infty) ;{ }^{s t} P^{s t} A P=-A\right\},
$$

with the $\mathbb{Z}_{2}$-gradation of $g l(\infty \mid \infty)$. Define $\operatorname{osp}(S)=\operatorname{osp}_{\underline{0}}(S) \oplus \operatorname{osp}_{\underline{1}}(S)$ by

$$
\operatorname{osp}_{\underline{i}}(S)=\bigoplus_{\underline{\mu+\underline{v}}=\underline{i}} \operatorname{osp}_{\underline{\mu}}(\infty \mid \infty) \otimes S_{\underline{v}} .
$$

We introduce a Lie supergroup $\operatorname{OSp}(S)$, which is generated by $\exp (A), A \in \operatorname{osp}_{\underline{0}}(S)$, as follows:

$$
\operatorname{OSp}(S)=\left\{A \in \operatorname{SGL}(S) ;{ }^{\tilde{s} t} P^{\tilde{s} t} A P=A^{-1}\right\}
$$

We define $\operatorname{OSp}(V)$ similarly. In the rest of this section we impose the restriction $t_{j}^{ \pm}=0$ for $j \equiv 0,3(\bmod 4)$. Put $\widetilde{S}=S l_{t_{j}^{ \pm}=0, j \equiv 0,3(\bmod 4)}$.

The OSp-STL hierarchy is a system of the Sato equations with a condition of symmetry: 


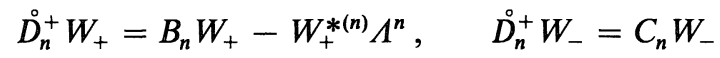

$$
\begin{aligned}
& D_{n}^{-} W_{+}=C_{n} W_{+}, \quad \stackrel{\circ}{n}_{n}^{-} W_{-}=C_{n} W_{-}-W_{-}^{*(n)} \Gamma^{-n}, \\
& \check{W}_{ \pm} \in \operatorname{OSp}(\widetilde{S}), \quad n \equiv 1,2(\bmod 4),
\end{aligned}
$$

where $W_{ \pm}=\sum_{j=0}^{\infty} \operatorname{diag}\left[w_{j}^{ \pm}(s)\right] \Lambda^{\mp j}, w_{j}^{ \pm}(s) \in \tilde{S}_{\underline{j}}, w_{0}^{+}(s)=1, \varepsilon\left(w_{0}^{-}(s)\right) \neq 0$ and $\stackrel{\circ}{D}_{n}^{ \pm}=\left.D_{n}^{ \pm}\right|_{\tilde{s}}$.

Theorem 3.1. If $W_{ \pm}$are the wave matrices of the OSp-STL hierarchy, then $\check{B}_{n}$, $\check{C}_{n} \in \operatorname{osp}_{\underline{n}}(\tilde{S})$ for $n \equiv 1,2(\bmod 4)$.

Proof. We see that

$$
{ }^{\tilde{s} t} P^{\tilde{s t}}\left(W_{+}^{*(n)} \Lambda^{n} W_{+}^{-1}\right) P=(-)^{n(n-1) / 2}\left(W_{+}^{*(n)} \Lambda^{n} W_{+}^{-1}\right)^{*(n)}
$$

by an easy calculation. Notice that ${ }^{\tilde{s} t}\left(A^{*(a)}\right)=(-)^{a}{ }^{s t} A$, where $A \in g l(S)_{\underline{a}}$. Hence we have

$$
{ }^{s t} P^{s t}\left(W_{+}^{*(n)} \Lambda^{n} W_{+}^{-1}\right) P=(-)^{n(n+1) / 2}\left(W_{+}^{*(n)} \Lambda^{n} W_{+}^{-1}\right) .
$$

Taking the (+)-part of (3.3), we see that $B_{n} \in \operatorname{osp}(\tilde{S})_{n}$ for $n \equiv 1,2(\bmod 4)$. For $C_{n}$ the proof is similar.

Q.E.D.

Let $\varepsilon\left(\check{W}_{ \pm}\right)=\left[\begin{array}{cc}W_{0 \overline{0}}^{ \pm} & 0 \\ 0 & W_{1 \frac{ \pm}{1}}^{ \pm}\end{array}\right]$. Then $W_{00}^{ \pm}$and $W_{11}^{ \pm}$are the wave matrices of the BTL hierarchy and the CTL hierarchy respectively for time evolutions $D_{4 n+2}^{+}(n \geq 0)$.

Put $\widetilde{B}_{1}=\left.B_{1}\right|_{t_{j}^{ \pm}=0, j>2}$ and $\widetilde{C}_{1}=\left.C_{1}\right|_{t_{j}^{ \pm}=0, j>2}$. Since $\check{B}_{1}, \check{C}_{1} \in \operatorname{osp}(\widetilde{S})_{\underline{1}}$, one has $\widetilde{\widetilde{B}}_{1}$, $\check{\widetilde{C}}_{1} \in \operatorname{osp}(\widetilde{S})_{1}$. Therefore the solution $u(s)$ of the STL equation (2.22) can be accompanied with the constraint

$$
u(s)=-u(-s)+\log (-1)^{s},
$$

where $\exp \left(\log (-1)^{s}\right)=(-1)^{s}$. We call the STL equation with the symmetry (3.4) the OSp-STL equation. Furthermore imposing the constraint $u(s+4 N)=u(s),(2.22)$ reduces to the following equations:

$$
\begin{aligned}
\tilde{D}_{1}^{+} \tilde{D}_{1}^{-} u(1) & =\exp (u(1))+\exp (u(2)-u(1)), \\
\tilde{D}_{1}^{+} \tilde{D}_{1}^{-} u(s) & =\exp (u(s)-u(s-1))+\exp (u(s+1)-u(s)), \quad 2 \leq s \leq 2 N-2, \\
\tilde{D}_{1}^{+} \tilde{D}_{1}^{-} u(2 N-1) & =\exp (u(2 N-1)-u(2 N-2))+\exp (-u(2 N-1)) .
\end{aligned}
$$

These equations coincide with the supper-Toda lattice equations corresponding to the Lie superalgebra $\operatorname{su}(2 N \mid 2 N+1)$ which are discussed in [1]. Putting $N=1$ in (3.5), we obtain the super-sine-Gordon equation

$$
\tilde{D}_{1}^{+} \tilde{D}_{1}^{-} u(1)=2 \cosh u(1) .
$$

The body part $f(1)=\varepsilon(u(1))$ satisfies the ordinary sine-Gordon equation

$$
\partial_{t_{2}^{+}} \partial_{t_{2}^{-}} f(1)=-2 \sinh 2 f(1) \text {. }
$$




\section{\$4. Am Explicit Representation of Solutions of the STL Hierarchy}

Put $\Phi_{+}=\exp \left(\sum_{j=0}^{\infty} t_{j}^{+} \Lambda^{j}\right)$ and $\Phi_{-}=\exp \left(\sum_{j=0}^{\infty} t_{j}^{-} \Gamma^{-j}\right)$.

Proposition 4. 1 . According to the $\mathrm{R}-\mathrm{H}$ decomposition

$$
\Phi_{+} A \Phi_{-}^{-1}=W_{+}^{-1} W_{-}
$$

for $A \in S G L(V)$, the STL hierarchy (2.23) is described by

$$
B_{n}=\left(W_{+}^{*(n)} \Lambda^{n} W_{+}^{-1}\right)_{+} \quad \text { and } \quad C_{n}=\left(W_{-}^{*(n)} \Gamma^{-n} W_{-}^{-1}\right)_{-} .
$$

Proof. From (4.1), we have

$$
W_{-}=W_{+} \Phi_{+} A \Phi_{-}^{-1}
$$

Note that $\Phi_{ \pm}$satisfy the equations

$$
D_{n}^{+} \Phi_{+}=\Lambda^{n} \Phi_{+} \quad \text { and } \quad D_{n}^{-} \Phi_{-}=\Gamma^{-n} \Phi_{-} .
$$

Operate $D_{n}^{+}$on (4.2). Then we have

$$
D_{n}^{+} W_{-}=\left(D_{n}^{+} W_{+}\right) \Phi_{+} A \Phi_{-}^{-1}+W_{+}^{*(n)} \Lambda^{n} \Phi_{+} A \Phi_{-}^{-1} .
$$

Multiply $W_{-}^{-1}$ each hand side of (4.3) from the right. Then we have

$$
\left(D_{n}^{+} W_{-}\right) W_{-}^{-1}=\left(D_{n}^{+} W_{+}\right) W_{+}^{-1}+W_{+}^{*(n)} \Lambda^{n} W_{+}^{-1} .
$$

Taking the (-)-part of (4.4), we obtain $D_{n}^{+} W_{+}=B_{n} W_{+}-W_{+}^{*(n)} \Lambda^{n}$. We can get other equations of (2.23) similarly.

Consider a matrix

$$
A=\left(\begin{array}{cc}
A_{00} & A_{01} \\
A_{10} & A_{11}
\end{array}\right)
$$

where $A_{i j} \in \operatorname{Mat}\left(\mathbb{N}^{c} \times \mathbb{N}^{c}: S_{i+j}\right)$, and $A_{00}$ and $A_{11}$ are invertible. Recall the definition of the superdeterminants of $A$ :

$$
\begin{aligned}
\text { s det } A & =\operatorname{det}\left(A_{00}-A_{01} A_{11}^{-1} A_{10}\right) / \operatorname{det} A_{11}, \\
\mathrm{~s}^{-1} \operatorname{det} A & =\operatorname{det}\left(A_{11}-A_{10} A_{00}^{-1} A_{01}\right) / \operatorname{det} A_{00} .
\end{aligned}
$$

It is known that $(\mathrm{s} \operatorname{det} A)\left(\mathrm{s}^{-1} \operatorname{det} A\right)=1$ (cf. [3]).

Theorem 4.2. Put $H=\Phi_{+} A \Phi_{-}^{-1}=\left(h_{i, j}\right)_{i, j \in \mathbb{Z}}$ for $A \in \operatorname{SGL}(V)$. And put $\tau(s)=$ $\mathrm{s} \operatorname{det}(H(s))$, where $H(s)=\left(h_{i, j}\right)_{i, j<s}$. Then, for the solution of the $R-H$ decomposition (4.1), we have

(i) $w_{1}^{+}(s)=D_{1}^{+} \log \tau(s)$,

(ii) $w_{0}^{-}(s)=1 /(\tau(s) \tau(s+1))$,

(iii) $w_{1}^{-}(s)=(-)^{s+1}\left(D_{1}^{-} \tau(s+1)\right) / \tau(s+1)^{2} \tau(s)$.

Proof. Let take the (-)-part of (4.2). Then we have $\left(W_{+} H\right)_{-}=0 . \quad$ From this we get the linear algebraic equation

$$
{ }^{t} \vec{w}_{+}(s) H(s)=-\left(\ldots h_{s, s-2}, h_{s, s-1}\right),
$$


where ${ }^{t} \vec{w}_{+}(s)=\left(\ldots w_{2}^{+}(s), w_{1}^{+}(s)\right) . \quad$ Take the check $(\ulcorner)$ of (4.5). Then we have

$$
\left(\vec{w}_{e}^{+}(s), \vec{w}_{0}^{+}(s)\right)\left(\begin{array}{cc}
H_{00}(s) & H_{01}(s) \\
H_{10}(s) & H_{11}(s)
\end{array}\right)=-\left(\vec{h}_{e}(s), \vec{h}_{o}(s)\right)
$$

where ${ }^{t} \vec{w}_{e}^{+}(s)=\left(\ldots w_{4}^{+}(s), w_{2}^{+}(s)\right),{ }^{t} \vec{w}_{o}^{+}(s)=\left(\ldots w_{3}^{+}(s), w_{1}^{+}(s)\right),{ }^{t} \vec{h}_{e}(s)=\left(\ldots h_{s, s-4}, h_{s, s-2}\right),{ }^{t} \vec{h}_{o}(s)=$ $\left(\ldots h_{s, s-3}, h_{s, s-1}\right)$ and $H_{\mu \nu}(s)=\left(h_{s-i, s-j}\right)_{\substack{i=\mu \\ \underline{j}=v i, j>0}}$. Multiplying $\left(\begin{array}{cc}1 & -H_{00}^{-1}(s) H_{01}(s) \\ 0 & 1\end{array}\right)$ to both sides of (4.6) from the right, we have

$$
{ }^{t} \vec{w}_{o}(s) \nabla(s)=-{ }^{t}\left(\alpha_{0, j}(s)\right)_{j<0},
$$

where

$$
\nabla(s)=\left(\alpha_{i j}(s)\right)_{i, j<0}=H_{11}(s)-H_{10}(s) H_{00}^{-1}(s) H_{01}(s)
$$

and

$$
{ }^{t}\left(\alpha_{0 j}(s)\right)_{j<0}={ }^{t} \vec{h}_{o}(s)-{ }^{t} \vec{h}_{e}(s) H_{00}^{-1}(s) H_{01}(s) .
$$

By Cramer's formula, we obtain a solution of (4.7),

$$
w_{1}^{+}(s)=-\sigma_{1}(s) / \sigma(s),
$$

where $\sigma_{1}(s)=\operatorname{det}\left(\begin{array}{c}\left(\alpha_{i j}(s)\right)_{i<-1} \\ t\left(\alpha_{0 j}(s)\right)_{j<0}\end{array}\right)$ and $\sigma(s)=\operatorname{det} \nabla(s)$. We rewrite (4.8) in such a way that

$$
w_{1}^{+}(s)=\frac{-\left(\sigma_{1}(s) / \operatorname{det} H_{00}(s)\right)}{\left(\sigma(s) / \operatorname{det} H_{00}(s)\right)} .
$$

\section{Lemma 4.4.}

$$
\sigma_{1}(s) / \operatorname{det} H_{00}(s)=D_{1}^{+}\left(\sigma(s) / \operatorname{det} H_{00}(s)\right) .
$$

Proof. From the construction of $H$

$$
D_{1}^{+} H=\Lambda H,
$$

we have the following relations:

$$
\begin{aligned}
& D_{1}^{+} H_{00}(s)=H_{10}(s), \\
& D_{1}^{+} H_{01}(s)=H_{11}(s), \\
& D_{1}^{+} H_{10}(s)=\Lambda_{\mathbf{N} c} H_{00}(s)+\left(\begin{array}{c}
0 \\
t \vec{h}_{e}(s)
\end{array}\right), \\
& D_{1}^{+} H_{11}(s)=\Lambda_{\mathbf{N}^{c}} H_{01}(s)+\left(\begin{array}{c}
0 \\
t \vec{h}_{o}(s)
\end{array}\right),
\end{aligned}
$$

where $\Lambda_{\mathbf{N}^{c}}=\left(\delta_{i+1, j}\right)_{i, j<0}$. Notice that $\sigma(s) / \operatorname{det} H_{00}(s)=\operatorname{det}\left(H_{00}^{-1}(s) \nabla(s)\right)$. From (4.12) we have

$$
D_{1}^{+}\left(H_{00}^{-1}(s) \nabla(s)\right)=H_{00}^{-1}(s)\left(\begin{array}{c}
0 \\
t\left(\alpha_{0 j}(s)\right)_{j<0}
\end{array}\right)
$$


$\operatorname{Put}\left(\zeta_{i j}(s)\right)_{i, j<0}=H_{00}^{-1}(s)$. Then one gets

$$
D_{1}^{+} \operatorname{det}\left(H_{00}^{-1}(s) \nabla(s)\right)=\sum_{k<0} \operatorname{det} \gamma_{k}(s)
$$

where

$$
\gamma_{k}(s)=\left(\begin{array}{c}
{ }^{t}\left(\zeta_{k-1}(s) \alpha_{0 j}(s)\right)_{j<0} \\
\left(\sum_{\rho<0} \zeta_{i \rho}(s) \alpha_{\rho j}(s)\right)_{j<0}
\end{array}\right) \begin{aligned}
& \cdots \text {-th row } \\
& \cdots i(\neq k) \text {-th row }
\end{aligned}
$$

Expanding each determinant of (4.14) along the $k$-th row, we have

$$
D_{1}^{+}\left(H_{00}^{-1}(s) D(s)\right)=\sum_{k<0} \sum_{j<0}(-)^{k+j} \zeta_{k-1}(s) \alpha_{0 j}(s) \Delta_{k j}(s),
$$

where $\Delta_{k j}(s)$ is a $(k, j)$-th minor determinant of $\gamma_{k}(s)$. One sees that

$$
\begin{aligned}
& \sum_{k<0} \sum_{j<0}(-)^{k+j} \zeta_{k-1}(s) \alpha_{0 j}(s) \Delta_{k j}(s) \\
& =\sum_{j<0} \alpha_{0 j}(s) \operatorname{det}\left[\begin{array}{lc}
\left(\sum_{l<0} \zeta_{i l}(s) \alpha_{l n}(s)\right)_{i<0} & \left(\zeta_{i-1}(s)\right)_{i<0} \\
n(\neq j) \text {-th column } & j \text {-th column }
\end{array}\right] \\
& =\sum_{j<0} \alpha_{0 j}(s) \operatorname{det}\left[\begin{array}{cc}
\left(\sum_{l<-1} \zeta_{i l}(s) \alpha_{l n}(s)\right)_{i<0} & \left(\zeta_{i-1}(s)\right)_{i<0} \\
n(\neq j) \text {-th column } & j \text {-th column }
\end{array}\right] \\
& =\sum_{j<0} \alpha_{0 j}(s) \operatorname{det}\left(\zeta_{\mu \nu}(s)\right)_{\mu, v<0} \operatorname{det}\left[\begin{array}{cc}
\left(\begin{array}{c}
\left(\alpha_{m n}(s)\right)_{m<-1} \\
0
\end{array}\right), & \left(\delta_{i,-1}\right)_{i<0} \\
n(\neq j) \text {-th column } & j \text {-th column }
\end{array}\right] \\
& =\operatorname{det}\left(\zeta_{\mu \nu}(s)\right)_{\mu, v<0} \sum_{j<0}(-)^{j+1} \alpha_{0 j}(s) \operatorname{det}\left(\alpha_{m n}(s)\right)_{m, n<-1} \neq j \\
& =\operatorname{det}\left(\zeta_{i j}(s)\right)_{i, j<0} \operatorname{det}\left(\alpha_{i j}(s)\right)_{\substack{i<1, i \neq-1 \\
j<0}} .
\end{aligned}
$$

This completes the proof of Lemma 4.4.

Let us return to the proof of Theorem 4.3. We see that

$$
w_{1}^{+}(s)=\frac{D_{1}^{+}\left(\operatorname{det} \nabla(s) / \operatorname{det} H_{00}(s)\right)}{\left(\operatorname{det} \nabla(s) / \operatorname{det} H_{00}(s)\right)} .
$$

Noting that $\tau(s)=\left(\operatorname{det} \nabla(s) / \operatorname{det} H_{00}(s)\right)^{-1}$, we get (i).

Let us show (ii). From (4.2) we have $\left(H W_{-}^{-1}\right)_{+}=1_{\mathbb{Z}}$, where $1_{\mathbb{Z}}$ is the unit matrix. We denote $W_{-}^{-1}=\sum_{j=0}^{\infty} \operatorname{diag}\left[u_{j}^{-}(s)\right] \Lambda^{j}$. Then one gets the linear equation

$$
\nabla(s+1) \vec{u}_{e}^{-}(s)=\left(\delta_{i,-1}\right)_{i<0},
$$

where $\vec{u}_{e}^{-}(s)=\left(u_{-2 j-2}^{-}(s+2 j+2)\right)_{j<0}$. By Cramer's formula, we get 


$$
u_{0}^{-}(s)=\frac{\tilde{\sigma}(s+1) / \operatorname{det} H_{00}(s+1)}{\operatorname{det}\left(\alpha(s+1)_{i j}\right)_{i, j<0} / \operatorname{det} H_{00}(s+1)},
$$

where $\tilde{\sigma}(s+1)=\operatorname{det}\left(\left(\alpha_{i j}(s+1)\right)_{\substack{i<0 \\ j<-1}}\left(\delta_{i,-1}\right)_{i<0}\right)$. We can easily verify that $\tilde{\sigma}(s+1)$ / $\operatorname{det} H_{00}(s+1)=\tau(s)$. From the fact that

$$
\operatorname{det} \nabla(s+1) / \operatorname{det} H_{00}(s+1)=1 / \tau(s+1)
$$

we have $u_{0}^{-}(s)=\tau(s) \tau(s+1)$. This completes the proof of (ii).

For a matrix $A=\sum_{j \in \mathbf{Z}} \operatorname{diag}\left[a_{j}(s)\right] \Lambda^{j}$, we put $(A)_{>0}=\sum_{j>0} \operatorname{diag}\left[a_{j}(s)\right] \Lambda^{j}$. From (4.2) one gets $\left(H W_{-}^{-1}\right)_{>0}=0$. Then we have the linear equation

$$
\nabla(s) \vec{u}_{o}^{-}(s)=-u_{0}^{-}(s)\left(\vec{h}_{o}(s)-H_{10}(s) H_{00}^{-1}(s) \vec{h}_{e}(s)\right),
$$

where $\vec{h}_{o}=\left(h_{s-2 i+1, s}\right)_{i>0}$ and $\vec{h}_{e}(s)=\left(h_{s-2 i, s}\right)_{i>0}$. We note that $\left(\alpha_{i 0}(s)\right)_{i<0}=\vec{h}_{o}(s)-$ $H_{10}(s) H_{00}^{-1}(s) \vec{h}_{e}(s)$. By Cramer's formula we see that

$$
u_{1}^{-}(s-1)=\frac{-u_{0}(s)\left(\tilde{\sigma}_{1}(s) / \operatorname{det} H_{00}(s)\right)}{\sigma(s) / \operatorname{det} H_{00}(s)}
$$

where $\tilde{\sigma}_{1}(s)=\operatorname{det}\left(\left(\alpha_{i j}(s)\right)_{\substack{i<0 \\ j<-1}}\left(\begin{array}{c}\left.\left(\alpha_{i 0}(s)\right)_{i<0}\right) . \text {. } \\ (-1)-\text { th column }\end{array}\right.\right.$ To show (iii), we prove the following lemma.

Lemma 4.5.

$$
D_{1}^{-}\left(\sigma(s) / \operatorname{det} H_{00}(s)\right)=(-)^{s+1}\left(\tilde{\sigma}_{1}(s) / \operatorname{det} H_{00}(s)\right) .
$$

Proof. From the equation $D_{1}^{-} H=H^{*} \Gamma^{-1}$, one gets the following relations:

$$
\begin{aligned}
& D_{1}^{-} H_{00}(s)=(-)^{s} H_{01}(s) \\
& D_{1}^{-} H_{01}(s)=(-)^{s}\left(H_{00}(s) \Lambda_{\mathbf{N}^{c}}^{-1}+\left[0, \vec{h}_{e}(s)\right]\right) \\
& D_{1}^{-} H_{10}(s)=(-)^{s+1} H_{11}(s) \\
& D_{1}^{-} H_{11}(s)=(-)^{s+1}\left(H_{10}(s) \Lambda_{\mathbf{N}^{c}}^{-1}+\left[0, \vec{h}_{o}(s)\right]\right)
\end{aligned}
$$

Notice that $\sigma(s) / \operatorname{det} H_{00}(s)=\operatorname{det}\left(\nabla(s) H_{00}^{-1}(s)\right)$. Then we have the relation

$$
D_{1}^{-}\left(\nabla(s) H_{00}^{-1}(s)\right)=(-)^{s+1}\left[0,\left(\alpha_{i 0}(s)\right)_{i<0}\right] H_{00}^{-1}(s) .
$$

Let us calculate $D_{1}^{-}$det $\left\{\left(\nabla(s) H_{00}^{-1}(s)\right\} . \quad\right.$ Let $\left(\beta_{i 0}(s)\right)_{i<0}=(-)^{s+1}\left(\alpha_{i 0}(s)\right)_{i<0}$. Then we have

$$
D_{1}^{-} \operatorname{det}\left\{\nabla(s) H_{00}^{-1}(s)\right\}=\sum_{k<0} \operatorname{det}\left[\begin{array}{cc}
\left(\sum_{\mu<0} \alpha_{i \mu}(s) \zeta_{\mu j}(s)\right)_{i<0},\left(\beta_{i 0}(s) \zeta_{-1, k}(s)\right)_{i<0} \\
\vdots & \vdots \\
j(\neq k) \text {-th column } & k \text {-th column }
\end{array}\right]
$$

Expand each determinant of (4.23) along the $k$-th column. Then we have 
the right hand side of $(4.23)=\sum_{k<0} \zeta_{-1, k}(s) \sum_{i<0}(-)^{i+k} \beta_{i 0}(s) \Delta_{i k}(s)$

$$
\begin{aligned}
& =\sum_{i<0} \beta_{i 0}(s) \operatorname{det}\left[\begin{array}{c}
t \\
\left.\sum_{\mu<0} \alpha_{k \mu}(s) \zeta_{\mu j}(s)\right)_{j<0} \\
{ }^{t}\left(\zeta_{-1 j}(s)\right)_{j<0}
\end{array}\right] \begin{array}{l}
\cdots \\
\cdots i \text {-th row }
\end{array} \\
& =\sum_{i<0} \beta_{i 0}(s) \operatorname{det}\left[\begin{array}{c}
t \\
\left.\sum_{\mu<-1} \alpha_{k \mu}(s) \zeta_{\mu j}(s)\right)_{j<0} \\
{ }^{(}\left(\zeta_{-1 j}(s)\right)_{j<0}
\end{array}\right] \begin{array}{l}
\cdots k(\neq i) \text {-th row } \\
\cdots i \text {-th row }
\end{array} \\
& =\sum_{i<0}(-)^{i+1} \beta_{i 0}(s) \operatorname{det}\left(\alpha_{k l}(s)\right)_{\substack{k<0 k \neq i \\
l<-1}} \operatorname{det}\left(\zeta_{\mu v}(s)\right)_{\mu, v<0} \\
& =\operatorname{det}\left[\left(\alpha_{i j}(s)\right)_{\substack{i<0 \\
j<-1}},\left(\beta_{i 0}(s)\right)_{i<0}\right] \operatorname{det}\left(\zeta_{i j}(s)\right)_{i, j<0} .
\end{aligned}
$$

This completes the proof of Lemma 4.5.

By Lemma $4.5, u_{1}(s-1)$ is represented as

$$
u_{1}(s-1)=\frac{(-)^{s+1} u_{0}(s)\left(D_{1}^{-}\left(\sigma(s) / \operatorname{det} H_{00}(s)\right)\right.}{\sigma(s) / \operatorname{det} H_{00}(s)} .
$$

Noting that $u_{1}^{-}(s)=-w_{1}^{-}(s) /\left(w_{0}^{-}(s) w_{0}^{-}(s+1)\right)$, we have (iii).

Q.E.D.

Proposition 4.6. Put $t_{j}^{ \pm}=0$ for $j \equiv 0,3(\bmod 4)$ and let $\check{A} \in \operatorname{OSp}(V)$ be decomposed as (4.1). Then $W_{ \pm}$in the right hand side are the wave matrices of the OSp-STL hierarchy.

Proof. Note that $\exp \left(\sum_{j \equiv 1,2(\bmod 4)} t_{j}^{+} \check{\Lambda}^{j}\right), \exp \left(\sum_{j \equiv 1,2(\bmod 4)} t_{j}^{-} \check{\Gamma}^{-j}\right) \in \operatorname{OSp}(\tilde{S})$. From the assumption of the Proposition 4.5, we have

$$
\left({ }^{\tilde{s} t} P^{\tilde{s} t} \breve{W}_{+}^{-1} P\right)\left({ }^{\tilde{s} t} P^{\tilde{s} t} \breve{W}_{-} P\right)=\exp \left(\sum_{j \equiv 1,2(\bmod 4)} t_{j}^{+} \check{\Lambda}^{j}\right) A \exp \left(\sum_{j \equiv 1,2(\bmod 4)} t_{j}^{-} \check{\Gamma}^{-j}\right) .
$$

By the uniqueness of the R-H decomposition, we have $\check{W}_{ \pm} \in \operatorname{OSp}(\tilde{S})$.

\section{References}

[1] Andreyev, V. A.: Supersymmetric generalized Toda lattice, Proc. Yurmala Seminar "Group Theoretical Methods in Physics", Markov, M. A. et al. ed., Vnu Science Press, 1986, 315-321.

[2] Chaichian, M. and Kulish, P. P.: On the method of inverse scattering problem and Bäcklund transformations for supersymmetric equations, Phys. Lett., 78B (1978), 413-416.

[3] DeWitt, B.: Supermanifolds, Cambridge UP., 1984.

[4] Ikeda, K.: A supersymmetric extension of the Toda lattice hierarchy, Lett. Math. Phys., 14 (1987), 321-328.

[5] Kac, V. G.: Lie superalgebras, Adv. Math., 26 (1977), 8-96.

[6] Leites, D. A.: Lie superalgebras, J. Soviet Math., 30(6) (1985), 2481-2512.

[7] Manin, Y. U. and Radul, A. O.: A supersymmetric extension of the Kadomtsev-Petviashvili hierarchy, Comm. Math. Phys., 98 (1985), 65-77.

[8] Mulase, M.: Solvability of the super KP hierarchy and a generalization of the Birkhoff decomposition, Inventiones Math., 98 (1988), 1-46. 
[9] Olshanetsky, M. A.: Supersymmetric two dimensional Toda lattice, Comm. Math. Phys., 88 (1983), 63-76.

[10] Takasaki, K.: Symmetries of the super KP hierarchy, to appear in Lett. Math. Phys.

[11] Ueno, K. and Takasaki, K.: Toda lattice hierarchy, Adv. Studies in Pure Math., 4 "Group Representations and Systems of Differential Equations", Kinokuniya 1984, 1-95.

[12] Ueno, K. and Yamada, H.: Super Kadomtsev-Petviashvili hierarchy and super Grassmann manifold, Lett. Math. Phys., 13 (1987), 59-68.

[13] - Supersymmetric extension of the Kadomtsev-Petviashvili hierarchy and universal super Grassmann manifold, Adv. Studies in Pure Math., 16 "Two-Dimensional Conformal Field Theory and Solvable Lattice Models", Kinokuniya 1988, 373-426.

[14] Ueno, K., Yamada, H., and Ikeda, K.: Algebraic study on the super-KP hierarchy and the orthosymplectic super-KP hierarchy, to appear in Comm. Math. Phys.

[15] Yamada, H.: Super Grassmann hierarchies-A multicomponent theory-Hiroshima Math. J., (1987), 373-394. 
